\title{
Doubly stochastic matrices and the quantum channels
}

\author{
H. K. DAS AND MD. KAISAR AHMED
}

\begin{abstract}
The main object of this paper is to study doubly stochastic matrices with majorization and the Birkhoff theorem. The Perron-Frobenius theorem on eigenvalues is generalized for doubly stochastic matrices. The region of all possible eigenvalues of $n$-by-n doubly stochastic matrix is the union of regular $(n-1)$ polygons into the complex plane. This statement is ensured by a famous conjecture known as the Perfect-Mirsky conjecture which is true for $n=1,2,3,4$ and untrue for $n=5$. We show the extremal eigenvalues of the Perfect-Mirsky regions graphically for $\mathrm{n}=1,2,3,4$ and identify corresponding doubly stochastic matrices. Bearing in mind the counterexample of Rivard-Mashreghi given in 2007, we introduce a more general counterexample to the conjecture for $\mathrm{n}=5$. Later, we discuss different types of positive maps relevant to Quantum Channels (QCs) and finally introduce a theorem to determine whether a QCs gives rise to a doubly stochastic matrix or not. This evidence is straightforward and uses the basic tools of matrix theory and functional analysis.
\end{abstract}

Mathematics Subject Classification 2010: 15B51, 15A18, 46A55, 46H05

Keywords: Stochastic Matrices, Doubly Stochastic Matrices, Eigenvalues, Eigenvectors, Linear Operators, Majoriation, Perfect-Mirsky Conjecture, Quantum Channel

\section{INTRODUCTION}

Stochastic and doubly stochastic matrices are mostly studied matrices for many years. Motivation has come from pure mathematics. The concept of stochastic matrices, a special type of nonnegative matrices, was introduced by Andrey Markov when he was working on the well-known mathematical system of the Markov chain in 1906. His intention of using these types of matrices was only for linguistic analysis and card shuffling. In 2017, the notion of steady-state is explored in connection with the long-run distribution, behavior of the Markov chain, and predictions based on Markov chains with more than two states are examined, followed by a discussion of the notion of absorbing Markov chains in Gagniuc [10]. Later Andrey Kolmogorov gave rise to developments of this type of matrices extending the possibilities of uses for continuous-time Markov processes [14]. 
As they were found to have a variety of applications in the fields like probability theory, statistics, mathematical finance, and linear algebra, as well as economics, engineering, computer science, population genetics, and QI; many of the researchers were intensely motivated by this. Prashanth et. al. [25] introduced some results using eigenvalues of signed graph using the number of vertices. In addition to these applications, stochastic modeling is an interesting and challenging domain of statistics and data science, for more details see Bhuiyan [3]. Moreover, the spectral properties of stochastic matrices appeared of great interest in the various domains of mathematics. The most facetious thing about the spectral properties of those matrices is the location of eigenvalues for different n. In 1936, Romanovsky [26] first tried to characterize the location of eigenvalues of stochastic matrices in the complex plane and he managed to find the points on the circumference of the unit circle where some eigenvalues of a different order of stochastic matrices may lie. But the problem of finding the possible area covering all the proper values of stochastic matrices was first suggested by Kolmogorov in 1938.

In 1946, Dmitriev et. al. [9] tried to mark the region, denoted by $\Theta_{n}$, given by the subset of the complex plane containing all possible eigenvalues of all $n$-by-n stochastic matrices. They managed to find that area in part. In 1938, Kolmogorov raised the question that what point of the circle of unity may serve as the characteristic root of a stochastic matrix. Later in 1946, Dmitriev and Dynkin [9] solved the problem completely for $n \leq 4$ and partially for $n>5$. On the basis of Dmitriv and Dynkin problem, Karpelevich [13] solved a similar problem completely and found the Karpelevich's region $\mathbb{K}_{n}$ in 1949. Karpelevič was able to solve the problem completely, but a new statement about it was made by Ito [11] in 1997. Some time later, Perfect et. al. [23] considered the same problem for double stochastic matrices, i.e., they tried to characterize the region $\omega_{n} \subset C$ containing all the eigenvalues of $\mathrm{n}$ by-n doubly stochastic matrices. They gave a conjecture about the possible region known as the Perfect-Mirsky conjecture. Recently, in 2015, Perfect-Mirsky Conjecture on the structure of the set of eigenvalues for all n-by-n doubly stochastic matrices in the four-dimensional case were studied by Levick J., Rajesh Pereira and David W. Kribs [19] and based on the analysis they made new conjectures for the 
general case. In this article, a detailed description of these results along with the diagrams of the regions are given to discuss matrices with extremal characteristic roots.

In the Quantum Information (QI) theory and Quantum system, a very important notion of quantum mechanics is the QCs are a special type of communication channel that delivers information from sender to receiver. The information conveyed by QCs is described by the quantum states of a quantum system. In addition, QCs can be regarded as a special type of positive maps on the matrix space. In this article, we discuss the idea of quantum information and the various types of positive maps concerning QCs. We also generalize the requirement that a QCs produces or does not produce a doubly stochastic matrix. An algebraic point of view, a QCs is a completely positive trace-preserving map that acts on space and gives the output of information to another space. Moreover, an euclidean formulation of relativistic quantum mechanics for systems of a finite number of degrees of freedom is considered in [27]. Based on unitarily, there may be two types of QCs: unital and non-unital. The initial QCs maintain the average of a quantum state. On the other hand, non-initial QC does not. As there is a connection between the positive trace-preserving initial maps and doubly stochastic matrices [5] there might have a connection between initial QCs and doubly stochastic matrices. In this paper, we will discuss the relationship between them. The remainder of this paper is divided into five sections. We first introduce some preliminary definitions and some relevant theorems as well as majorization illustrating with an example. In section three, we state the Perron-Frobenius theorem of nonnegative matrices. For showing the region of characteristic roots of such matrices, we first recall a theorem of Karpelevich regarding stochastic matrices. Then, we discuss the Perfect-Mirsky conjecture and show the matrices with extremal roots graphically in the star shaped region. In section four, we introduce a more general form of counterexamples for Perfect-Mirsky conjecture and compare it with that of previously found Rivard-Mashreghi counterexample for $n=5$. Section five is devoted to the quantum channels and discusses the connection between doubly stochastic matrices and trace preserving initial positive maps. We finally generalize the 
condition, whether a quantum channel gives rise to doubly stochastic matrix or not by a theorem.

\section{MATHEMATICAL BACKGROUND}

A special type of real matrices whose all entries are positive, called positive matrices. The stochastic matrices are the simplest form of positive matrices in which certain conditions are implemented. There are two simple types of positive matrices row (column) stochastic matrices and doubly stochastic matrices. Each entry of such matrices represents a probability. In this section, we discuss doubly stochastic matrices and the famous theorem of Birkhoff is regarding those types of matrices. We define majorization for one dimensional and multidimensional case and formulate a lemma connecting doubly stochastic matrices and majorization. (For more details, see Armandnejad et. al. [1].)

DEFINITION 1. A matrix $\mathrm{A}=\left(\mathrm{a}_{\mathrm{ij}}\right) \in M_{n}(\mathfrak{R})$ is called row (column) stochastic if the sum of each of its rows (columns) equals to 1 . That is,

(i) $a_{i j} \geq 0$, for $i, j=1, \ldots \ldots \ldots, n$.

(ii) $\sum_{j=1}^{n} a_{i j}=1$, for $i=1, \ldots \ldots \ldots, n\left(\sum_{i=1}^{n} a_{i j}=1\right.$ for $\left.j=1, \ldots \ldots \ldots, n\right)$.

The set of all $n \times n$ row (column) stochastic matrices is denoted by $\Omega_{n}^{\text {row }}\left(\Omega_{n}^{\text {column }}\right)$.

DEFINITION 2. A matrix $A \in M_{n}(\mathfrak{R})$ is called doubly stochastic if the sum of each of its rows and columns equals to 1 i. e. $(i) a_{i j} \geq 0$, for $i, j=1, \ldots n$, and (ii) $\sum_{j=1}^{n} a_{i j}=\sum_{i=1}^{n} a_{i j}=1$, for $i, j=1, \ldots n$.

Doubly stochastic matrices are also known as Bistochastic matrices, Schur transformation. The set of all $n \times n$ doubly stochastic matrices is called Birkhoff polytope and is denoted by $\Omega_{n}$. A special example of doubly stochastic matrices are the permutation matrices, the square matrices whose entries are all either 0 or 1 and which contain exactly a 1 in each row and each column.

THEOREM 1. (Mehlum [22]) The set of all $n \times n$ doubly stochastic matrices is convex. 
THEOREM 2. (Birkhoff [2]) The set $\Omega_{n}$ of doubly stochastic matrices of size nby- $n$ is the convex hull of the $n-b y-n$ permutation matrices.

To get a better understanding of the Birkhoff theorem of doubly stochastic matrices and their importance for this paper, we present an example to illustrate how the doubly stochastic matrices can be expressed as the convex combination of permutation matrices. This is illustrated by the following example:

EXAMPLE 1. Illustrate the doubly stochastic matrix $\left(\begin{array}{ccc}\frac{1}{\mathbf{1 0}} & \frac{3}{\mathbf{1 0}} & \frac{3}{5} \\ \frac{3}{5} & \frac{1}{10} & \frac{3}{10} \\ \frac{3}{\mathbf{1 0}} & \frac{3}{5} & \frac{1}{\mathbf{1 0}}\end{array}\right)$ for

Birkhoff theorem.

Proof: To illustrate the Birkhoff theorem, set $A_{0}=\left(\begin{array}{ccc}\frac{1}{10} & \frac{3}{10} & \frac{3}{5} \\ \frac{3}{5} & \frac{1}{10} & \frac{3}{10} \\ \frac{3}{10} & \frac{3}{5} & \frac{1}{10}\end{array}\right)$, then we have the following:

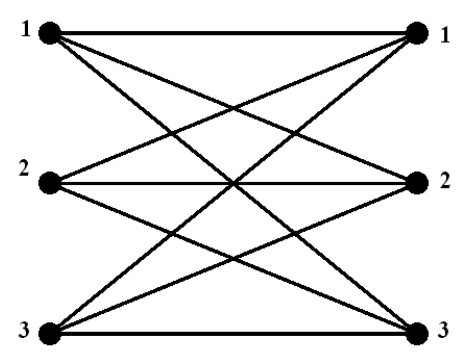

Fig. 1: Bipartite graph associated to $A_{0}$ matrix

A perfect matching is $\{(1,1),(2,3),(3,2)\}$ and the corresponding permutation matrix is $P_{0}=\left(\begin{array}{lll}1 & 0 & 0 \\ 0 & 0 & 1 \\ 0 & 1 & 0\end{array}\right)$. The smallest entry of $A_{0}$ corresponding to the non-zero entries of $P_{0}$ is $\alpha_{0}=\frac{1}{10}$ (If $\alpha_{0}=1$, then we would have $A_{0}=P_{0}$ ), then we get the following doubly stochastic matrix, 


$$
A_{1}=\frac{1}{1-\alpha_{0}}\left(A_{0}-\alpha_{0} P_{0}\right)=\frac{10}{9}\left[\left(\begin{array}{ccc}
\frac{1}{10} & \frac{3}{10} & \frac{3}{5} \\
\frac{3}{5} & \frac{1}{10} & \frac{3}{10} \\
\frac{3}{10} & \frac{3}{5} & \frac{1}{10}
\end{array}\right)-\left(\begin{array}{ccc}
\frac{1}{10} & 0 & 0 \\
0 & 0 & \frac{1}{10} \\
0 & \frac{1}{10} & 0
\end{array}\right)\right]==\left(\begin{array}{ccc}
0 & \frac{1}{3} & \frac{2}{3} \\
\frac{2}{3} & \frac{1}{9} & \frac{2}{9} \\
\frac{1}{3} & \frac{5}{9} & \frac{1}{9}
\end{array}\right) \text { and we }
$$

have the bipartite graph associated to $A_{1}$ in the following:

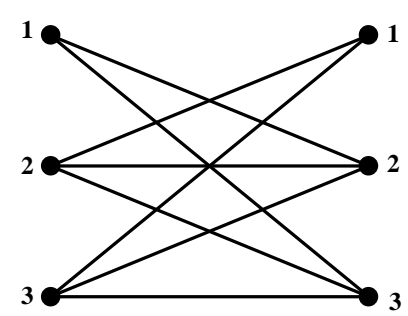

Fig. 2: Bipartite graph associated to $A_{1}$ matrix

A perfect matching is $\{(1,2),(2,1),(3,3)\}$, and the corresponding permutation matrix is $P_{1}=\left(\begin{array}{lll}0 & 1 & 0 \\ 1 & 0 & 0 \\ 0 & 0 & 1\end{array}\right)$. Then we have $A_{2}=\frac{1}{1-\alpha_{1}}\left(A_{1}-\alpha_{1} P_{1}\right)=\left(\begin{array}{ccc}0 & \frac{1}{4} & \frac{3}{4} \\ \frac{5}{8} & \frac{1}{8} & \frac{1}{4} \\ \frac{3}{8} & \frac{5}{8} & 0\end{array}\right) \quad$ of $\quad A_{1}$ corresponding to the non-zero entries of $P_{1}$ is $\alpha_{1}=\frac{1}{9}$. Bipartite graph associated to $A_{2}$ is

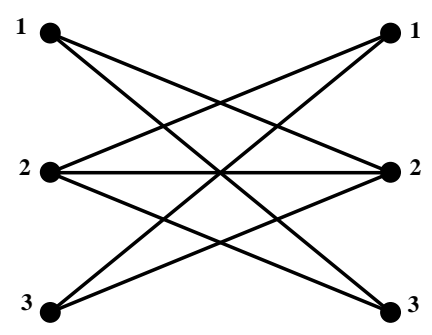

Fig. 3: Bipartite graph associated to $\mathrm{A}_{2}$ matrix 
A perfect matching is $\{(1,3),(2,2),(3,1)\}$ and the corresponding permutation matrix is $P_{2}=\left(\begin{array}{lll}0 & 0 & 1 \\ 0 & 1 & 0 \\ 1 & 0 & 0\end{array}\right)$. We then get $A_{3}=\frac{1}{1-\alpha_{2}}\left(A_{2}-\alpha_{2} P_{2}\right)=\left(\begin{array}{ccc}0 & \frac{2}{7} & \frac{5}{7} \\ \frac{5}{7} & 0 & \frac{2}{7} \\ \frac{2}{7} & \frac{5}{7} & 0\end{array}\right)$ by choosing the smallest entry of $A_{2}$ and then Bipartite graph associated to $A_{3}$ is following.

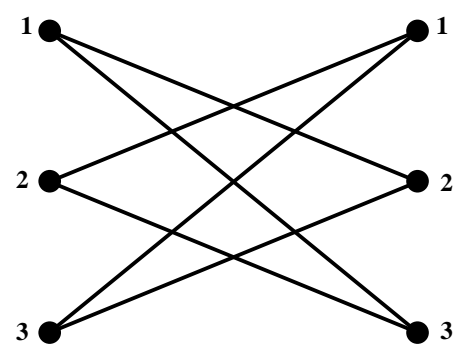

Fig. 4: Bipartite graph associated to $A_{3}$ matrix

A perfect matching is $\{(1,3),(2,1),(3,2)\}$ and the corresponding permutation matrix is $P_{3}=\left(\begin{array}{lll}0 & 0 & 1 \\ 1 & 0 & 0 \\ 0 & 1 & 0\end{array}\right)$. The smallest entry of $A_{3}$ to the non-zero entries of $P_{3}$ is $\alpha_{3}=\frac{5}{7}$, then we get $A_{4}=\frac{1}{1-\alpha_{2}}\left(A_{2}-\alpha_{2} P_{2}\right)=\left(\begin{array}{lll}0 & 1 & 0 \\ 0 & 0 & 1 \\ 1 & 0 & 0\end{array}\right)=P_{4}$. Thus, $A_{3}=\alpha_{3} P_{3}+\left(1-\alpha_{3}\right) A_{4}=\frac{5}{7} P_{3}+\frac{2}{7} P_{4}, \quad A_{4}=P_{4}$ $A_{2}=\alpha_{2} P_{2}+\left(1-\alpha_{2}\right) A_{3}=\frac{1}{8} P_{2}+\frac{5}{8} P_{3}+\frac{2}{8} P_{4}$ $A_{1}=\alpha_{1} P_{1}+\left(1-\alpha_{1}\right) A_{2}=\frac{1}{9} P_{1}+\frac{1}{9} P_{2}+\frac{5}{9} P_{3}+\frac{2}{9} P_{4}$, and finally we get $A_{0}=\alpha_{0} P_{0}+\left(1-\alpha_{0}\right) A_{1}=\frac{1}{10} P_{0}+\frac{1}{10} P_{1}+\frac{1}{10} P_{2}+\frac{5}{10} P_{3}+\frac{2}{10} P_{4}$. Therefore, we finally have 
$\left(\begin{array}{ccc}\frac{1}{10} & \frac{3}{10} & \frac{3}{5} \\ \frac{3}{5} & \frac{1}{10} & \frac{3}{10} \\ \frac{3}{10} & \frac{3}{5} & \frac{1}{10}\end{array}\right)=\frac{1}{10}\left(\begin{array}{ccc}1 & 0 & 0 \\ 0 & 0 & 1 \\ 0 & 1 & 0\end{array}\right)+\frac{1}{10}\left(\begin{array}{ccc}0 & 1 & 0 \\ 1 & 0 & 0 \\ 0 & 0 & 1\end{array}\right)+\frac{1}{10}\left(\begin{array}{lll}0 & 0 & 1 \\ 0 & 1 & 0 \\ 1 & 0 & 0\end{array}\right)+\frac{5}{10}\left(\begin{array}{lll}0 & 0 & 1 \\ 1 & 0 & 0 \\ 0 & 1 & 0\end{array}\right)+\frac{2}{10}\left(\begin{array}{lll}0 & 1 & 0 \\ 0 & 0 & 1 \\ 1 & 0 & 0\end{array}\right)$,

where $\alpha_{0}+\alpha_{1}+\alpha_{2}+\alpha_{3}+\alpha_{4}=1$.

We now describe how two vectors $X$ and $Y$ are related to each other by majorization and doubly stochastic matrices. Normally, it measures which of the vectors of $X$ or $Y$ is "more or less spread out". For example, in economics, the majorization is used to compare the income distribution of two groups of the population.

DEFINITION 3. Let $X=\left(x_{1}, x_{2}, \ldots \ldots \ldots, x_{n}\right)$ and $Y=\left(y_{1}, y_{2}, \ldots \ldots \ldots, y_{n}\right)$ be the $n$-tuples of real numbers. Then we say that $X$ is majorized by $Y$, written $X \prec Y$, if $\sum_{i=1}^{k} x_{i}^{\downarrow}=\sum_{i=1}^{k} y_{i}^{\downarrow} ; 1 \rightleftarrows \leq k \leq n$ and $\sum_{i=1}^{n} x_{i}=\sum_{i=1}^{n} y_{i}$. The notation $X^{\downarrow}$ means that the entries of $X$ are arranged in descending order. We present now a real life example for explaining the notion of majorization in our own way as follows:

EXAMPLE 2. Consider two Cricket teams in Oklahoma State University, Stillwater, OK, USA namely, Cowboy cricket club $A$, and Cowboy cricket club $B$. Both teams scored 150 runs in a 20-20 over match, resulted in a tie. But someone may try to identify which team was better. The question is: can we really identify properly which team was better?

Two teams played 20 overs, scored 150, but they scored different runs in different phase of the game. Let us divide the phases in terms of overs. That is, the scoring rate in each over is not equally distributed. We re-arrange the runs scored in each over in an ascending sequence. $x_{i}$ and $y_{i}$ represent the runs in the $i^{\text {th }}$ over of team $A$ and $B$ respectively. Now we compute the relative runs of the $i^{\text {th }}$ over to be sum of the $i^{\text {th }}$ smallest overs divided by total runs. We represent this numbers by $\overline{x_{i}}$ and $\overline{y_{i}}$ respectively. We also define $n_{i}$ to be the proportion of overs having runs $x_{i}\left(y_{i}\right)$ or less, such that $n_{n}$ denotes the whole overs. Then we plot the pairs $\left(n_{i}, \overline{x_{i}}\right)$ and $\left(n_{i}, \overline{y_{i}}\right)$, $i=1, \ldots \ldots \ldots, 20$ in the same axes of coordinates. We add a straight line which 
represents the overs where scores are at level. The associated graph shown in the following:

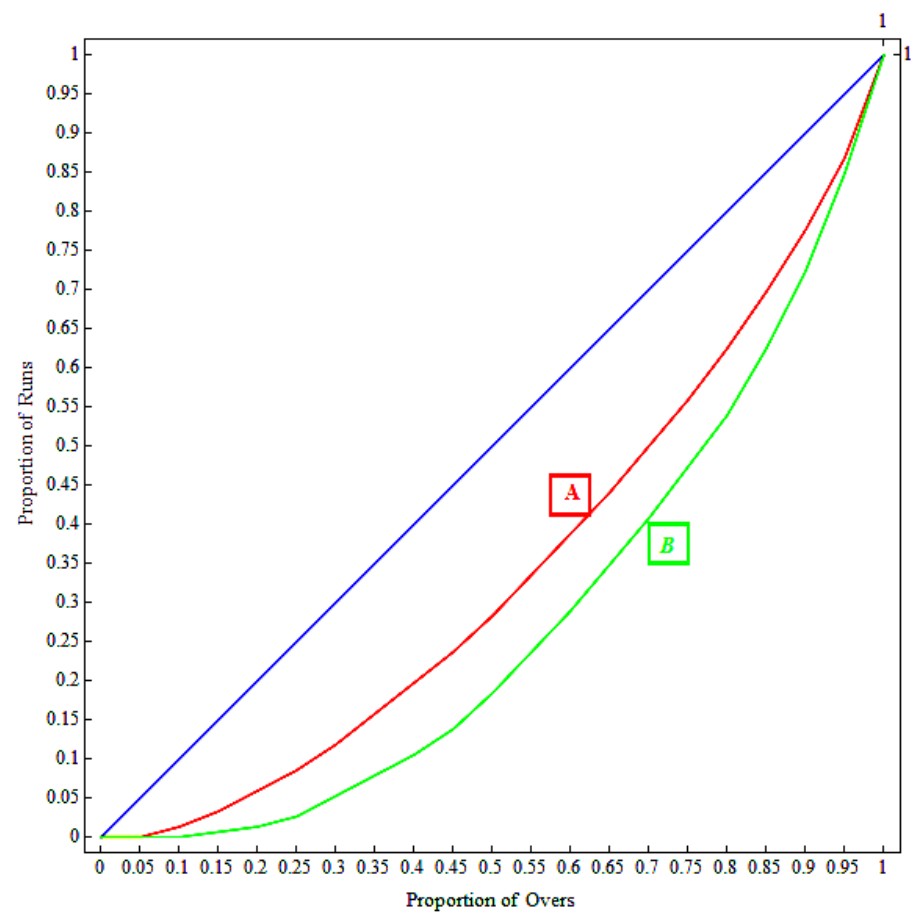

Fig. 5: Comparison of two Cowboy Cricket Clubs over by over

we observe that the graph of $A$ is less convex than the graph of $B$ or $B$ spread out more than $A$ Using this concept, we can conclude that $A$ is more evenly distributed than $B$ or $A$ is a better team than $B$ Although the idea of majorization comes from the comparison of two distinct objectives such as their income, height or other things, this concept is extended for a vector of more objects or points. But the problem for solving the comparison for a multidimensional array is more complicated than that of the single vector case. For a multidimensional case or simply for a matrix, the majorization is characterized differently using the concept of doubly stochastic matrices. 
THEOREM 3. Let $\boldsymbol{X}=\{\boldsymbol{x}: \boldsymbol{X} \prec \boldsymbol{Y}\}$. Then the set $X$ is the convex hull of points generated by permuting the points of $Y$. (Marshall [20])

DEFINITION 4. Let $A$ and $B$ be two $m \times n$ real matrices. Then we say that $A$ is majorized by $B$ if there exists at least one doubly stochastic matrix $D$ of order $m \times m$ such that $A=B D$.

The definition above is equivalent to saying that $A$ is row (column) majorized by $B$ if each row (column) of $A$ is majorized by the corresponding row (column) of $B$. For more details characterization of row or column majorization one can see Armandnejad et. al. [1], and Dahl [8]. Linking up theorem 3 and definitions above, we have the following relation between majorization and doubly stochastic matrices.

LEMMA 1. Let $x, y \in \mathfrak{R}_{\geq 0}^{n}$. Then the followings are equivalent:

$$
\begin{aligned}
& \text { 1. } x<y \\
& \text { 2. } x \in \operatorname{Conv}\{y\} \\
& \text { 3. } x=y D \text { for some doubly stochastic matrix } D \in \Omega_{n} .
\end{aligned}
$$

\section{REGIONS OF CHARACTERISTIC ROOTS OF DOUBLY STOCHASTIC MATRICES}

One of the most important theorems in matrix analysis is the Perron-Frobenius theorem, a theorem regarding the eigenvalues and corresponding eigenvectors of nonnegative matrices. This theorem also has a similar assertion for different classes of non-negative matrices. In 2012, Cheng et.al. [4], provide a simple proof for the Perron-Frobenius theorem concerned with positive matrices using a homotopy technique. In this section, we give our observation of the Perron-Frobenius theorem in the case of stochastic and doubly stochastic matrices. We discuss the PerfectMirsky conjecture for doubly stochastic matrices and show the extremal characteristic roots of $\omega_{n}$ for $n \geq 2$.

THEOREM 4. (Perron-Frobenius theorem, (Cheng et. Al. [4])) If $A=\left(a_{i j}\right)$ is a real $n \times n$ non-negative matrix, then 
(i) $A$ has a non-negative eigenvalue $\lambda$ which is equal to the spectral radius of $A$

(ii) There is a unique eigenvector corresponding to $\lambda$ whose all entries are non-negative.

In our case, the simple observation of Perron-Frobenius theorem is that

(i) Every stochastic and doubly stochastic matrix has spectral radius 1 .

(ii) The eigenvector corresponding to the spectral radius is $(1,1, \ldots \ldots, 1)^{T}$.

\section{Perfect-Mirsky Conjecture.}

It was observed that the subset of $C$ which may contain the eigenvalues of an $n \times n$ doubly stochastic matrix is a subset of the characteristic region of an $n \times n$ stochastic matrix. Also, the characteristic region of $(n-1) \times(n-1)$ doubly stochastic matrices is a subset of the characteristic region of $n \times n$ doubly stochastic matrices.

DEFINITION 5. Let $A$ be an $n \times n$ doubly stochastic matrix and $\lambda$ be any complex number inside the unit circle. Then the set of eigenvalues of $A$ consists of the collection of all $\lambda$ 's if there exists a $v$ corresponding to $\lambda$ such that $A v=\lambda v$.

$$
\text { i.e, } \omega_{n}=\left\{\lambda \in C: \exists A \in \Omega_{n}, A v=\lambda v\right\} \text {. }
$$

DEFINITION 6. The region $\prod_{n}$ is the closed region whose boundary is the regular $n$-gon circumscribed in the unit circle with vertices at $\left\{1, e^{\frac{i 2 \pi}{n}}, e^{\frac{i 4 \pi}{n}}, \ldots \ldots \ldots, e^{\frac{i 2(n-1) \pi}{n}}\right\}$. That is,

$$
\Pi_{n}=\operatorname{Conv}\left\{e^{\frac{i 2 \pi k}{n}}: 0 \leq k \leq n-1\right\} .
$$

CONJECTURE 1. (Perfect and Mirsky [23] ) $\omega_{n}=\bigcup_{i=1}^{n} \Pi_{i}$.

The Perfect-Mirsky conjecture is trivially true for $n=1,2$ and Perfect and Mirsky proved the conjecture for $n=3$. In 2015 Levick et. al. [19], Levick [18] in his Ph.D. 
thesis, and latter in 2016 Levick [17] proved this conjecture for $n=4$, and the conjecture is false for $n=5$. Still for the higher values of $n$, the conjecture is unknown.

\section{Matrices with Extremal Eigenvalues}

According to the Perfect-Mirsky conjecture the region $\omega_{n}$ is the union of the $n$-gons in the unit circle. For $n=1$, the matrix $A=[1]$ has the requirement of doubly stochastic matrices and its only eigenvalue is 1 , located at the point $(1,0)$ on the arc of the unit circle. To discuss the matrices with extremal characteristic roots for $n \geq 2$, we first define doubly stochastic circulant matrix slightly modifying the definition of circulant matrix in Kra and Simanca [15] as follows:

DEFINITION 7. Let us consider a row vector

$$
v=\left\{\left(v_{0}, v_{1}, \ldots \ldots \ldots, v_{n-1}\right) \in \mathfrak{R}^{n}: v_{i} \in[0,1], \sum_{i=0}^{n-1} v_{i}=1\right\} .
$$

and define a shift operator $\sigma\left(v_{0}, v_{1}, \ldots \ldots, v_{n-1}\right)=\left(v_{n-1}, v_{0}, \ldots \ldots, v_{n-2}\right)$.

Then the doubly stochastic circulant matrix $A=\operatorname{cir}(v)$ associated to the vector $v \in$ $\mathfrak{R}^{n}$ is the $n \times n$ matrix whose rows are generated by the shift operator $\sigma$ defined on $v$; its $i^{\text {th }}$ row is $\sigma^{i-1}(v), i=1, \ldots \ldots \ldots, n$ :

$$
A=\left(\begin{array}{ccccccc}
v_{0} & v_{1} & \ldots & \ldots & \ldots & v_{n-2} & v_{n-1} \\
v_{n-1} & v_{0} & \ldots & \ldots & \ldots & v_{n-3} & v_{n-2} \\
\vdots & \vdots & & & & \vdots & \vdots \\
\vdots & \vdots & & & & \vdots & \vdots \\
\vdots & \vdots & & & & & \vdots \\
v_{2} & v_{3} & \ldots & \ldots & \ldots & v_{0} & v_{1} \\
v_{1} & v_{2} & \ldots & \ldots & \ldots & v_{n-1} & v_{0}
\end{array}\right)
$$

PROPOSITION 1. For $n \geq 3$, the $n$-gon described in definition 6 corresponds $\frac{n}{2}$ (when $n$ is even) or $\frac{n+1}{2}$ (when $n$ is odd) doubly stochastic circulant matrices.

The sides connected with the point 1 represent a trace non-zero doubly stochastic circulant matrix with at most two non-zero adjacent entries. The other sides of the 
n-gon represent trace zero doubly stochastic circulant matrices with at most two nonzero adjacent entries. For example, in the heptagon $\left(\prod_{7}\right)$ for $n=7$, we get

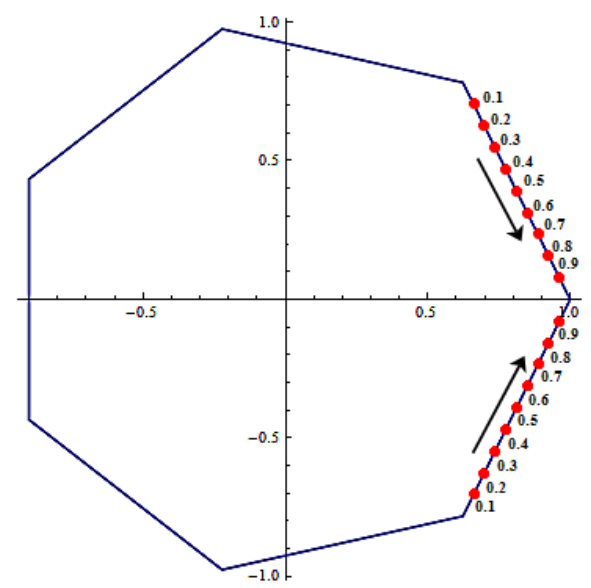

Fig. 6: The region $\prod_{7}$.

where the lines connected to the point 1 is represented by the trace non-zero doubly stochastic circulant matrix

$$
\left(\begin{array}{ccccccc}
t & 1-t & 0 & 0 & 0 & 0 & 0 \\
0 & t & 1-t & 0 & 0 & 0 & 0 \\
0 & 0 & t & 1-t & 0 & 0 & 0 \\
0 & 0 & 0 & t & 0 & 0 & 0 \\
0 & 0 & 0 & 0 & t & 1-t & 0 \\
0 & 0 & 0 & 0 & 0 & t & 1-t \\
1-t & 0 & 0 & 0 & 0 & 0 & t
\end{array}\right) ; t \in[0,1] .
$$

\section{A. The Region $\omega_{2}$}

The region $\omega_{2}=\prod_{1} \cup \prod_{2}$ consists of the line joining the points 1 and $e^{i \pi}$. The $2 \times 2$ doubly stochastic circulant matrix with eigenvalues located on this line is of the form $A=\left(\begin{array}{cc}t & 1-t \\ 1-t & t\end{array}\right), t \in[0,1]$. Note that, all roots on this line are extremal and as $t$ varies we get 


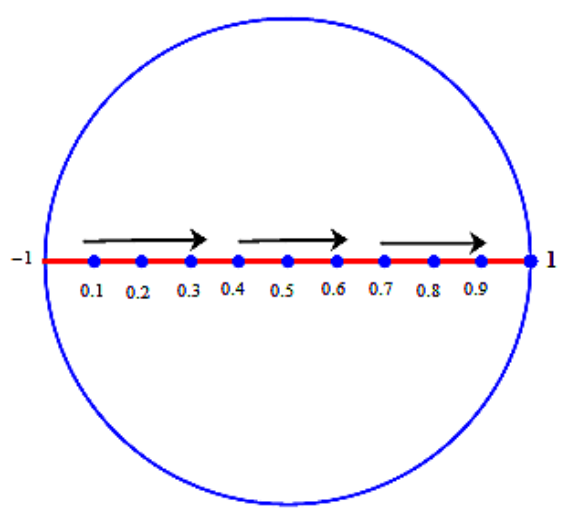

Fig. 7: Extremal roots of $\omega_{2}$

\section{B. The Region $\omega_{3}$}

The region $\omega_{3}=\prod_{1} \cup \prod_{2} \cup \prod_{3}$ consists of a regular triangle with vertices at $1, e^{\frac{i 2 \pi}{3}}$ and $e^{\frac{i 4 \pi}{3}}$ and of the closed interval $\left[-1,-\frac{1}{2}\right]$.

(1) The $3 \times 3$ doubly stochastic trace non-zero circulant matrix with extremal eigenvalues located on the lines joining the point 1 to the points $e^{\frac{i 2 \pi}{3}}$ and $e^{\frac{i 4 \pi}{3}}$ is of the form $A_{31}=\left(\begin{array}{ccc}t & 1-t & 0 \\ 0 & t & 1-t \\ 1-t & 0 & t\end{array}\right)$ and the following figure shows the location of the roots as $t$ varies:

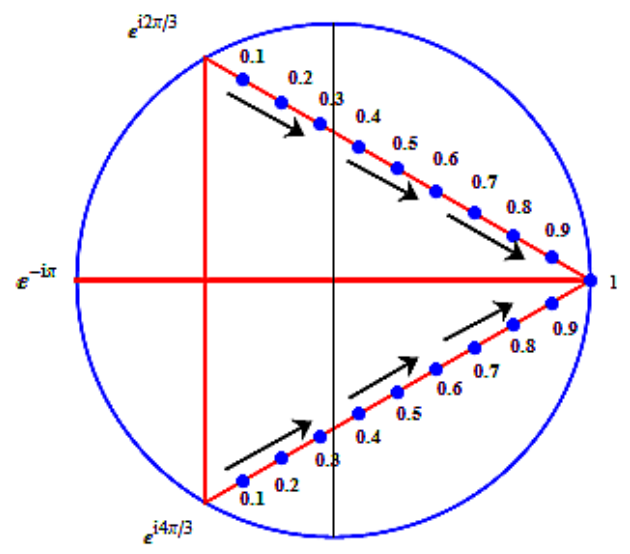

Fig. 8: Extremal roots of $\omega_{3}$ between $\left\langle 1, e^{\frac{i 2 \pi}{3}}\right\rangle$ and $\left\langle 1, e^{\frac{i 4 \pi}{3}}\right\rangle$. 
(2) The $3 \times 3$ doubly stochastic trace zero circulant matrix with extremal eigenvalues located on the lines joining the points $e^{\frac{i 2 \pi}{3}}$ and $e^{\frac{i 4 \pi}{3}}$ is of the form:

$$
A_{32}=\left(\begin{array}{ccc}
0 & t & 1-t \\
1-t & 0 & t \\
t & 1-t & 0
\end{array}\right) .
$$

Following figure shows the location of the roots as $t$ varies:

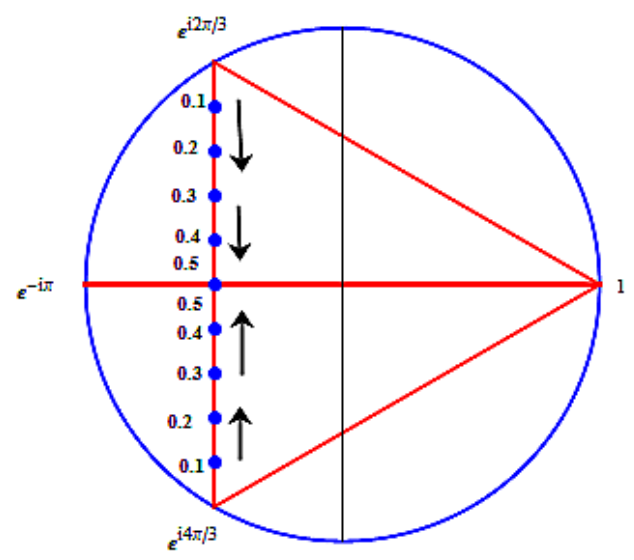

Fig. 9: Extremal roots of $\omega_{3}$ between $\left\langle e^{\frac{i 2 \pi}{3}}, e^{\frac{i 4 \pi}{3}}\right\rangle$

(3) For the closed interval $\left[-1,-\frac{1}{2}\right]$ this line has the characteristic polynomial multiplied by the factor $\lambda-1$ to the characteristic polynomial of $A$ in $2 \times 2$ case. So the $3 \times 3$ matrix is obtained simply by adding a new column and a new row with $3^{\text {rd }}$ entry 1 to the matrix $A$. The $3 \times 3$ doubly stochastic matrix with extremal eigenvalues located $\left[-1,-\frac{1}{2}\right]$ on the real line is of the form $A_{33}=\left(\begin{array}{ccc}t & 1-t & 0 \\ 1-t & t & 0 \\ 0 & 0 & 1\end{array}\right)$, and following figure shows the location of the roots as $t$ varies: 


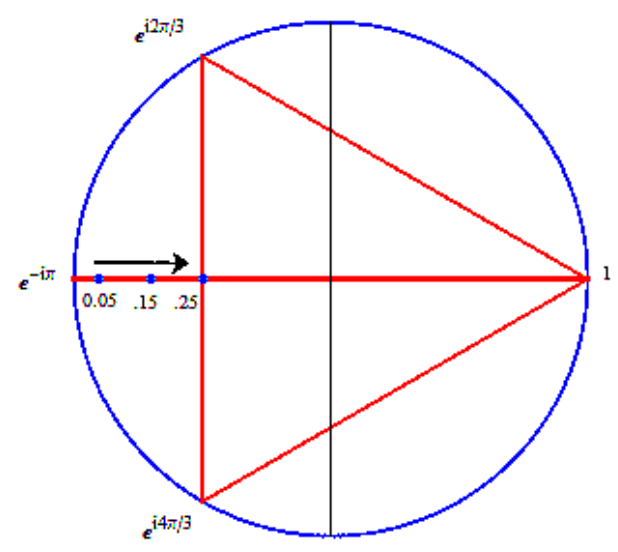

Fig. 10: Extremal roots of $\omega_{3}$ between $\left[-1,-\frac{1}{2}\right]$

\section{The Region $\omega_{4}$}

The region $\omega_{4}$ intersects the unit circle at six points, $1, e^{\frac{i \pi}{2}}, e^{\frac{i 2 \pi}{3}}, e^{\frac{i 4 \pi}{3}}, e^{i \pi}$ and $e^{\frac{i 3 \pi}{2}}$, consisting of $\prod_{1} \cup \prod_{2} \cup \prod_{3} \cup \prod_{4}$.

(1) The $4 \times 4$ doubly stochastic trace non-zero circulant matrix with extremal eigenvalues located on the lines joining the points 1 to the points $e^{\frac{i 2 \pi}{3}}$ and $e^{\frac{i 4 \pi}{3}}$ is of the form $A_{41}=\left(\begin{array}{cccc}t & 1-t & 0 & 0 \\ 0 & t & 1-t & 0 \\ 0 & 0 & t & 1-t \\ 1-t & 0 & 0 & t\end{array}\right)$, and following figure shows the location of roots as $t$ varies 


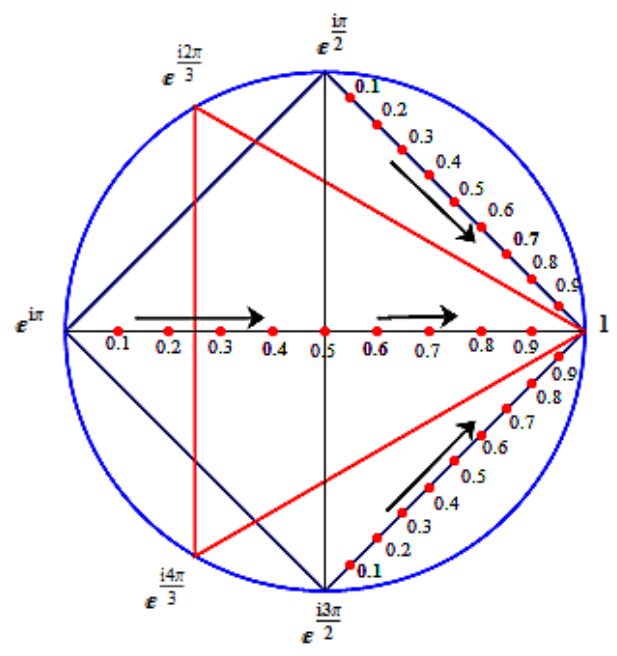

Fig. 11: Extremal roots of $\omega_{4}$ between $\left\langle 1, e^{\frac{i \pi}{2}}\right\rangle\left\langle 1, e^{\frac{i \pi}{2}}\right\rangle$ and $\left\langle 1, e^{\frac{i 3 \pi}{2}}\right\rangle$

(2) Following figure shows the location of the root as $t$ varies:

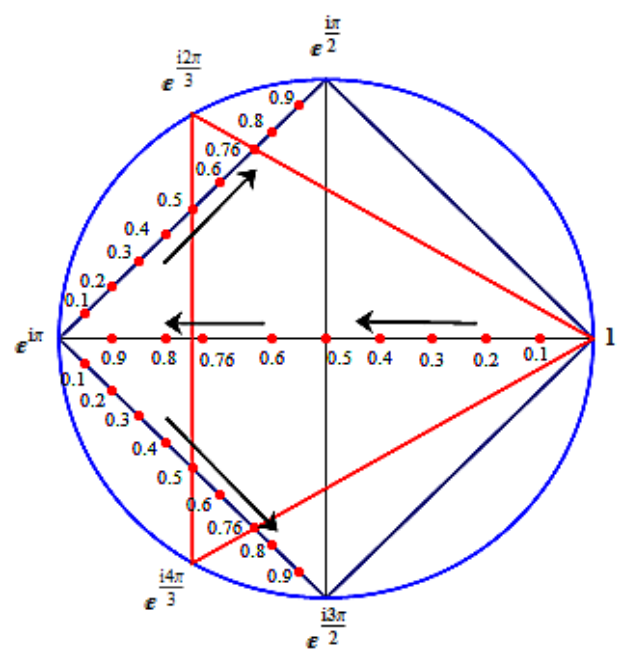

Fig. 12: Extremal roots of $\omega_{4 .}$ between $\left\langle e^{i \pi}, e^{\frac{i \pi}{2}}\right\rangle$ and $\left\langle e^{i \pi}, e^{\frac{i 3 \pi}{2}}\right\rangle$ 
Where, the $4 \times 4$ doubly stochastic trace zero circulant matrix with extremal eigenvalues located on the lines joining the points $e^{i \pi}$ to the points $e^{\frac{\mathrm{i} 2 \pi}{3}}$ and $e^{\frac{\mathrm{i} 4 \pi}{3}}$ is of the form

$$
A_{42}=\left(\begin{array}{cccc}
0 & t & 1-t & 0 \\
0 & 0 & t & 1-t \\
1-t & 0 & 0 & t \\
t & 1-t & 0 & 0
\end{array}\right)
$$

(3) For the lines joining the point 1 to the points $e^{\frac{i 2 \pi}{3}}$ and $e^{\frac{i 4 \pi}{3}}$ respectively, these lines have the characteristic polynomial multiplied by the factor $\lambda-1$ to the characteristic polynomial of the matrix $A_{31}$ in $3 \times 3$ case. So the $4 \times 4$ doubly stochastic trace non-zero matrix is obtained simply by adding a new column and a new row with $4^{\text {th }}$ entry 1 to the matrix $A_{31}$. The $4 \times 4$ doubly stochastic matrix with extremal eigenvalues are of the form $A_{43}=\left(\begin{array}{cccc}t & 1-t & 0 & 0 \\ 0 & t & 1-t & 0 \\ 1-t & 0 & t & 0 \\ 0 & 0 & 0 & 1\end{array}\right)$, and following figure shows the location of the roots as $t$ varies:

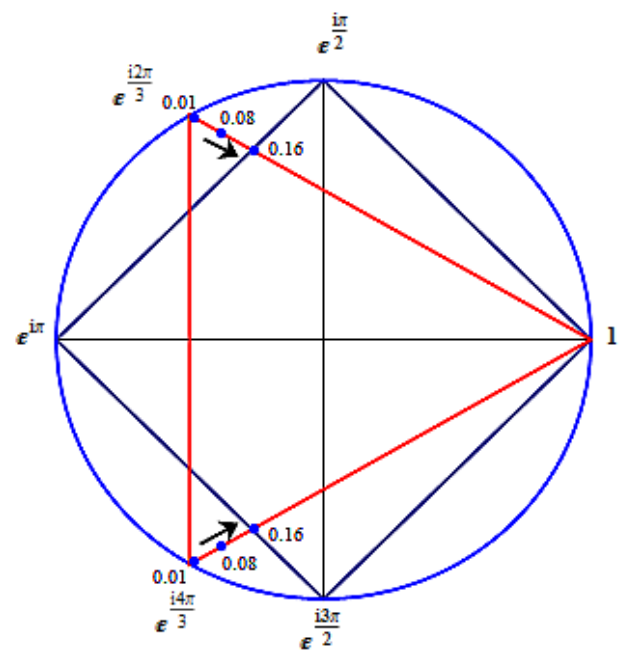

Fig. 13: Extremal roots of $\omega_{4}$ between $\left\langle 1, e^{\frac{i 2 \pi}{3}}\right\rangle$ and $\left\langle 1, e^{\frac{i 4 \pi}{3}}\right\rangle$ 
(4) For the line joining the points $e^{\frac{\mathrm{i} 2 \pi}{3}}$ and $e^{\frac{\mathrm{i} 4 \pi}{3}}$ has the characteristic polynomial multiplied by the factor $\lambda-1$ to the characteristic polynomial of the matrix $A_{32}$ in $3 \times 3$ case. Thus $4 \times 4$ doubly stochastic matrix is obtained simply by adding a new column and a new row with $4^{\text {th }}$ entry 1 to the matrix $A_{32}$. The $4 \times 4$ doubly stochastic matrix with extremal eigenvalues is of the form $A_{44}=\left(\begin{array}{cccc}0 & t & 1-t & 0 \\ 1-t & 0 & t & 0 \\ t & 1-t & 0 & 0 \\ 0 & 0 & 0 & 1\end{array}\right)$, and following figure shows the location of the roots as $t$ varies:

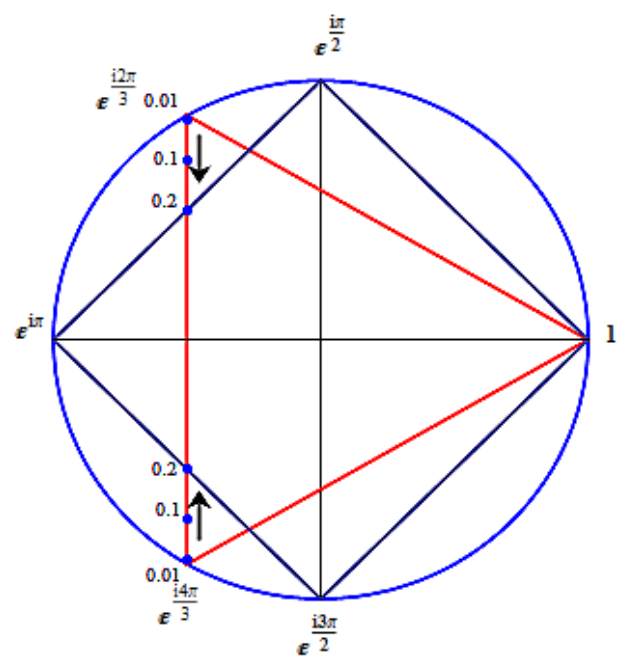

Fig. 14: Extremal roots of $\omega_{4}$. between $\left\langle e^{\frac{i 2 \pi}{2}}, e^{\frac{i 4 \pi}{3}}\right\rangle$

\section{COUNTEREXAMPLE FOR $\boldsymbol{n}=\mathbf{5}$}

In this section, we give a more general structure of counterexample of the PerfectMirsky conjecture for $n=5$ than that of Rivard-Mashreghi and at the end, we produce some particular cases for counterexample to make reasonable comparison.

\section{Rivard-Mashreghi Counterexample.}

In 2007, Mashregi et. al. [21] found a counterexample, showing that $\bigcup_{i=1}^{5} \prod i \underset{\neq}{\subset} \omega_{5}$. 


$$
P_{t}=\left(\begin{array}{ccccc}
0 & 0 & 0 & 1 & 0 \\
0 & 0 & t & 0 & 1-t \\
0 & t & 1-t & 0 & 0 \\
0 & 1-t & 0 & 0 & t \\
1 & 0 & 0 & 0 & 0
\end{array}\right)
$$

They numerically showed that the matrix has an eigenvalue for $t \in[0.5-\varepsilon, 0.5+\varepsilon]$ outside the Perfect-Mirsky region. They showed that the matrix

$$
P_{0.5}=\left(\begin{array}{ccccc}
0 & 0 & 0 & 1 & 0 \\
0 & 0 & 0.5 & 0 & 0.5 \\
0 & 0.5 & 0.5 & 0 & 0 \\
0 & 0.5 & 0 & 0 & 0.5 \\
1 & 0 & 0 & 0 & 0
\end{array}\right)
$$

has two complex eigenvalues $\lambda \approx 0.28 \pm 0.76 i$, lying outside the region $\omega_{5}$.

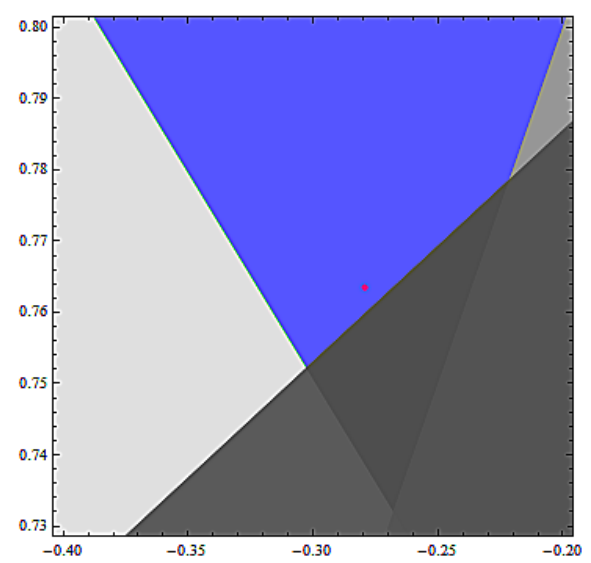

Fig. 15: The Rivard-Mashregi Counterexample for $\omega_{5}$ 


\section{New Counterexamples for $\boldsymbol{n}=\mathbf{5}$.}

We propose a new matrix of the form

$$
\left(\begin{array}{ccccc}
r & 0 & 0 & 1-r & 0 \\
0 & s & t & 0 & 1-s-t \\
0 & t & 1-s-t & 0 & s \\
0 & 1-s-t & s & 0 & t \\
1-r & 0 & 0 & r & 0
\end{array}\right)
$$

For $r \in[0-\varepsilon, 0+\varepsilon], s \in[0-\eta, 0+\eta]$ and $t \in[0.5-v, 0.5+v]$, the matrix has some eigenvalues outside the Perfect-Mirsky region.

CASE (I). For $r=0, s=0$, and $t=\frac{1}{2}$, we get the Rivard-Mashreghi counterexample from our proposed structure of matrix.

CASE (II). Again, from equation (1), for $r=0.001, s=0.001, t=0.494$, we have the matrix

$$
A=\left(\begin{array}{ccccc}
0.001 & 0 & 0 & 0.999 & 0 \\
0 & 0.001 & 0.494 & 0 & 0.505 \\
0 & 0.494 & 0.505 & 0 & 0.001 \\
0 & 0.505 & 0.001 & 0 & 0.494 \\
0.999 & 0 & 0 & 0.001 & 0
\end{array}\right)
$$

has two roots outside the region $\omega_{5}$, and geometrically we have

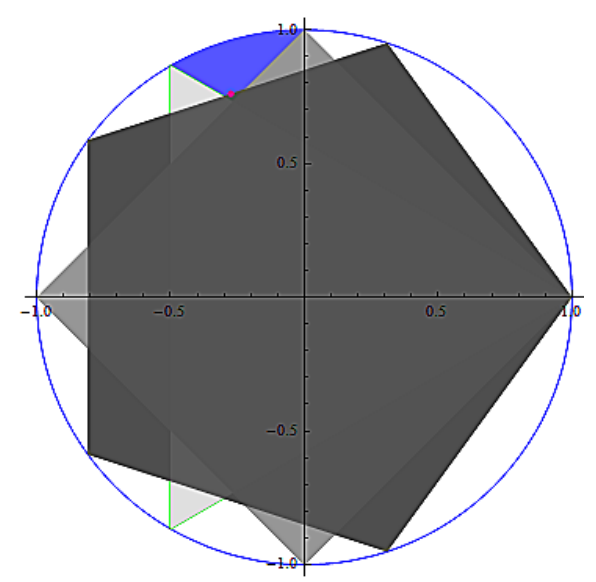

Fig. 16: Eigenvalue location of the matrix A 
CASE (II) MATHEMATICAL EXPLAINATION. The characteristic polynomial of $A$ is

$\operatorname{Det}(\lambda I-A)=-\lambda^{5}+0.507 \lambda^{4}+0.243519 \lambda^{3}+0.492774 \lambda^{2}+0.00480328 \lambda-0.248096$.

One root of the polynomial is 1 , so we factor out the term $\lambda-1$ and let the remaining part be denoted by $f(\lambda)=-\lambda^{4}-0.493 \lambda^{3}-0.249481 \lambda^{2}+0.243292 \lambda+0.248096$. The characteristic polynomial has two more real roots and two complex roots which are (using the Mathematica software) approximately $\lambda_{1} \approx 0.642647, \lambda_{2} \approx-0.587367$, and $a \pm i b \approx-0.27414 \pm 0.762959 i$. Let $\lambda_{1}, \lambda_{2} \in \Re$ and $a \pm i b$ be the other roots of the equation $f(\lambda)=-\lambda^{4}-0.493 \lambda^{3}-0.249481 \lambda^{2}+0.243292 \lambda+0.248096=0$. Then the sum of the roots is

$$
\lambda_{1}+\lambda_{2}+2 a=-0.493
$$

and the product of the roots is

$$
\lambda_{1} \lambda_{2}\left(a^{2}+b^{2}\right)=-0.248096
$$

Now we show that the roots $a \pm i b$ to the forbidden region denoted by $\Delta$.

We find that $f(0.642646)>0$, and $f(0.642648)<0$. Hence, by the Intermediate Value, we have $\lambda_{1} \in[0.642646,0.642648]$. In a similar way, $f(-0.587366)>0$, and $f(-0.587368)<0$ and we find $\lambda_{2} \in[-0.587366,-0.587368]$.

Hence, by (3), we get $a \in[-0.27413,-0.27415]$, and, by (4), we get $b \in$ [0.762958,0.762960],

This is same to saying that

$$
a+i b \in[-0.27413,-0.27415] \times[0.762958,0.762960]=Z .
$$

Now, the equation of the line joining the points $(-1,0)$ and $(0,1)$ is $y-x-1=0$, the equation of the line joining the points $(1,0)$ and $e^{\frac{i 4 \pi}{3}}$ is $\sqrt{3} y+x-1=0$, and the equation of the line joining the points $e^{\frac{i 2 \pi}{5}}$ and $e^{\frac{i 4 \pi}{5}}$ is $\frac{x-\operatorname{Cos}\left(\frac{2 \pi}{5}\right)}{\operatorname{Cos}\left(\frac{4 \pi}{5}\right)-\operatorname{Cos}\left(\frac{2 \pi}{5}\right)}-\frac{y-\operatorname{Sin}\left(\frac{2 \pi}{5}\right)}{\operatorname{Sin}\left(\frac{4 \pi}{5}\right)-\operatorname{Sin}\left(\frac{2 \pi}{5}\right)}$.

Let $F(x, y)=y-x-1=0, G(x, y)=\sqrt{3} y+x-1=0$, 


$$
H(x, y)=\frac{x-\operatorname{Cos}\left(\frac{2 \pi}{5}\right)}{\operatorname{Cos}\left(\frac{4 \pi}{5}\right)-\operatorname{Cos}\left(\frac{2 \pi}{5}\right)}-\frac{y-\operatorname{Sin}\left(\frac{2 \pi}{5}\right)}{\operatorname{Sin}\left(\frac{4 \pi}{5}\right)-\operatorname{Sin}\left(\frac{2 \pi}{5}\right)}
$$

Then it is easy to verify that $F(x, y)>0, G(x, y)>0, H(x, y)>0$ for the four extreme corner points of $Z$ and ensure that the rectangle $Z$ lies in the forbidden region $\Delta$.

CASE (III). For $r=0, s=0.01, t=0.52$, from equation (4.5.1), we have the matrix

$$
B=\left(\begin{array}{ccccc}
0 & 0 & 0 & 1 & 0 \\
0 & 0.01 & 0.52 & 0 & 0.47 \\
0 & 0.52 & 0.505 & 0 & 0.01 \\
0 & 0.47 & 0.01 & 0 & 0.52 \\
1 & 0 & 0 & 0 & 0
\end{array}\right)
$$

has two roots $\lambda \approx-0.298683 \pm 0.755402$ outside the region $\omega_{5}$.

Note that, the following matrices $A$ and $B$ are a Convex Combination of 3 Permutation Matrices, where

$$
A=\left(\begin{array}{ccccc}
0.001 & 0 & 0 & 0.999 & 0 \\
0 & 0.001 & 0.494 & 0 & 0.505 \\
0 & 0.494 & 0.505 & 0 & 0.001 \\
0 & 0.505 & 0.001 & 0 & 0.494 \\
0.999 & 0 & 0 & 0.001 & 0
\end{array}\right), \text { and } B=\left(\begin{array}{ccccc}
0 & 0 & 0 & 1 & 0 \\
0 & 0.01 & 0.52 & 0 & 0.47 \\
0 & 0.52 & 0.505 & 0 & 0.01 \\
0 & 0.47 & 0.01 & 0 & 0.52 \\
1 & 0 & 0 & 0 & 0
\end{array}\right) \text {. }
$$

Proof: Let us consider the matrix $B$. We set it as

$$
B_{0}=\left(\begin{array}{ccccc}
0 & 0 & 0 & 1 & 0 \\
0 & 0.01 & 0.52 & 0 & 0.47 \\
0 & 0.52 & 0.47 & 0 & 0.01 \\
0 & 0.47 & 0.001 & 0 & 0.52 \\
1 & 0 & 0 & 0 & 0
\end{array}\right)
$$


The bipartite graph associated to $B_{0}$ is the following:

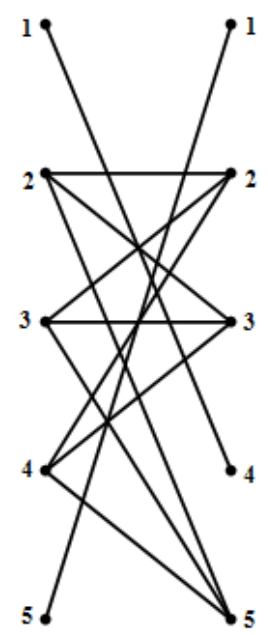

Fig. 17: Bipartite graph associated to $B_{0}$ matrix

A perfect matching is $(1,4),(2,3),(3,2),(4,5),(5,1)$ and the corresponding permutation matrix is

$$
P_{0}=\left(\begin{array}{lllll}
0 & 0 & 0 & 1 & 0 \\
0 & 0 & 1 & 0 & 0 \\
0 & 1 & 0 & 0 & 0 \\
0 & 0 & 0 & 0 & 1 \\
1 & 0 & 0 & 0 & 0
\end{array}\right)
$$

The smallest entry of $B_{0}$ corresponding to the non-zero entries of $P_{0}$ is $\alpha_{0}=0.52$.

Then, we get

$$
B_{1}=\frac{1}{1-\alpha_{0}}\left(B_{0}-\alpha_{0} P_{0}\right)=\frac{1}{0.48}\left(\begin{array}{ccccc}
0 & 0 & 0 & 0.48 & 0 \\
0 & 0.01 & 0 & 0 & 0.47 \\
0 & 0 & 0.47 & 0 & 0.01 \\
0 & 0.47 & 0.01 & 0 & 0 \\
0.48 & 0 & 0 & 0 & 0
\end{array}\right)=\left(\begin{array}{ccccc}
0 & 0 & 0 & 1 & 0 \\
0 & \frac{1}{48} & 0 & 0 & \frac{47}{48} \\
0 & 0 & \frac{47}{48} & 0 & \frac{1}{48} \\
0 & \frac{47}{48} & \frac{1}{48} & 0 & 0 \\
1 & 0 & 0 & 0 & 0
\end{array}\right)
$$


The bipartite graph associated to $B_{1}$ is the following:

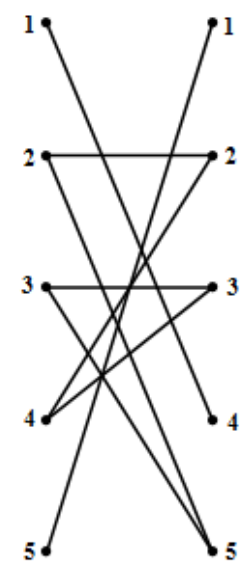

Fig. 18: Bipartite graph associated to $B_{l}$ matrix

A perfect matching is $(1,4),(2,2),(3,5),(4,3),(5,1)$ and the corresponding permutation matrix is

$$
P_{1}=\left(\begin{array}{lllll}
0 & 0 & 0 & 1 & 0 \\
0 & 1 & 0 & 0 & 0 \\
0 & 0 & 0 & 0 & 1 \\
0 & 0 & 1 & 0 & 0 \\
1 & 0 & 0 & 0 & 0
\end{array}\right)
$$

The smallest entry of $B_{1}$ corresponding to the non-zero entries of $P_{1}$ is $\alpha_{1}=\frac{1}{48}$.

Then, we get

$$
B_{2}=\frac{1}{1-\alpha_{1}}\left(B_{1}-\alpha_{1} P_{1}\right)=\frac{48}{0.47}\left(\begin{array}{ccccc}
0 & 0 & 0 & \frac{47}{48} & 0 \\
0 & 0 & 0 & 0 & \frac{47}{48} \\
0 & 0 & \frac{47}{48} & 0 & 0 \\
0 & \frac{47}{48} & 0 & 0 & 0 \\
\frac{47}{48} & 0 & 0 & 0 & 0
\end{array}\right)=\left(\begin{array}{ccccc}
0 & 0 & 0 & 1 & 0 \\
0 & 0 & 0 & 0 & 1 \\
0 & 0 & 1 & 0 & 0 \\
0 & 1 & 0 & 0 & 0 \\
1 & 0 & 0 & 0 & 0
\end{array}\right)=P_{2}
$$


We now go backward, and then

$$
\begin{gathered}
B_{1}=\alpha_{1} P_{1}+\left(1-\alpha_{1}\right) B_{2} \\
B_{0}=\alpha_{0} P_{0}+\left(1-\alpha_{0}\right) B_{1} \\
=\alpha_{0} P_{0}+\left(1-\alpha_{0}\right)\left\{\alpha_{1} P_{1}+\left(1-\alpha_{1}\right) P_{2}\right\} \\
=0.52 P_{0}+0.01 P_{1}+0.47 P_{2} .
\end{gathered}
$$

Finally we have

$$
\left(\begin{array}{ccccc}
0 & 0 & 0 & 1 & 0 \\
0 & 0.01 & 0.52 & 0 & 0.47 \\
0 & 0.52 & 0.47 & 0 & 0.01 \\
0 & 0.47 & 0.001 & 0 & 0.52 \\
1 & 0 & 0 & 0 & 0
\end{array}\right)=0.52\left(\begin{array}{lllll}
0 & 0 & 0 & 1 & 0 \\
0 & 0 & 1 & 0 & 0 \\
0 & 1 & 0 & 0 & 0 \\
0 & 0 & 0 & 0 & 1 \\
1 & 0 & 0 & 0 & 0
\end{array}\right)+0.01\left(\begin{array}{lllll}
0 & 0 & 0 & 1 & 0 \\
0 & 1 & 0 & 0 & 0 \\
0 & 0 & 0 & 0 & 1 \\
0 & 0 & 1 & 0 & 0 \\
1 & 0 & 0 & 0 & 0
\end{array}\right)+0.47\left(\begin{array}{lllll}
0 & 0 & 0 & 1 & 0 \\
0 & 0 & 0 & 0 & 1 \\
0 & 0 & 1 & 0 & 0 \\
0 & 1 & 0 & 0 & 0 \\
1 & 0 & 0 & 0 & 0
\end{array}\right) \text {, }
$$

as a consequence of Birkhoff theorem which says that $B_{0}$ is a convex combination of 3 permutation matrices.

\section{Comparing our counterexamples to that of Rivard-Mashreghi.}

We note that the Rivard-Mashreghi counterexample is a convex combination of just two permutation matrices; but our new counterexamples are convex combination of three permutation matrices.

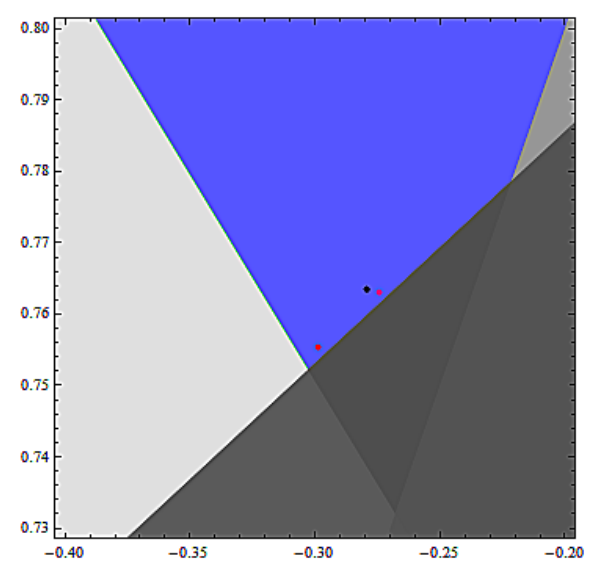

Fig. 19: Comparison of counterexamples of case (i), (ii) and (iii) 
Comparing our counterexamples to that of Rivard-Mashreghi, we see that for more terms in the convex combination, the eigenvalues are more close to the real boundary of $\omega_{5}$. Since the region $\omega_{n}$ approaches the full circle, the forbidden region inside the circle becomes smaller. And since each vertex on the unit circle represents a permutation matrix, so if there exists any counterexample to the Perfect-Mirsky conjecture for higher values of $n$, they are expected to be a convex combination of fewer permutation matrices. For deeper understanding, one can see Levick [18].

\section{QUANTUM CHANNELS}

In this section, we introduce a theorem to determine whether a QCs gives rise to a doubly stochastic matrix or not.

DEFINITION 8. A symmetric matrix $A$ is called positive semidefinite if $x^{*} A x \geq$ 0 for all column vector $x$ in $\mathbb{C}^{\mathrm{n}}$ (or, $x$ in $\mathfrak{R}^{n}$ for the real matrices). If $x^{*} A x>0$, then $A$ is said to be positive definite. We note that the eigenvalues of all positive semidefinite matrices are nonnegative whereas the eigenvalues of all positive definite matrices are positive.

DEFINITION 9. A positive semidefinite matrix $\rho$ of $n \times n$ complex entries is called a density matrix if $\operatorname{Tr}(\rho)=1$. Mathematically, density matrices can be formulated as $\rho=\sum p_{i}\left|\psi_{i}\right\rangle\left\langle\psi_{i}\right|$ (see details Das[7] for some remarkable results depending on density matrices). Thus, any general density matrix is a convex combination of pure state density matrices; hence density matrices are generally considered to be as mixed states.

DEFINITION 10. Let $M_{n}$ be a set of $n \times n$ complex matrices. A positive map is a linear map defined as $\Phi: M_{n_{1}} \rightarrow M_{n_{2}}$ such that it maps positive semidefinite matrix $X \in M_{n_{1}}$ into a positive semidefinite matrix $\Phi(X) \in M_{n_{2}}$. We recall that if the matrix $X$ is positive, then we have

$$
\langle\psi|X| \psi\rangle \geq 0
$$


for all $\psi \in C^{n}$, and the map $\Phi$ is positive if it satisfies

$$
\langle\psi|\Phi(|\phi\rangle\langle\phi|)| \psi\rangle \geq 0 .
$$

for $\varphi \in C^{n_{1}}$ and $\psi \in C^{n_{2}}$.

DEFINITION 11. Let $i d_{n}: M_{n} \rightarrow M_{n}$ be an identity map for which $i d_{n}(X)=X$ for any $X \in M_{n}$. Then a linear map $\Phi: M_{n_{1}} \rightarrow M_{n_{2}}$ is said to be $n$-positive if $\Phi \otimes$ $i d_{n}: M_{n_{1}} \otimes M_{n} \rightarrow M_{n_{2}} \otimes M_{n}$ is positive.

DEFINITION 12. A linear map $\Phi: M_{n_{1}} \rightarrow M_{n_{2}}$ is said to be completely positive if it is $n$-positive for all $n=1,2, \ldots$.

we note that due to Kraus et. al. [16], any completely positive map $\Phi: M_{n_{1}} \rightarrow M_{n_{2}}$ can be expressed as

$$
\Phi(X)=\sum_{i} K_{i} X K_{i}^{*}
$$

The representation (11) is called the Kraus decomposition of the map $\Phi$, and the operators $\left\{K_{i}\right\}$ are called the Kraus operators of $\Phi$. Every Kraus operator is an $n_{1} \times n_{2}$ matrix such that (11) holds for all $X \in M_{n_{1}}$. We recall that similar to the definition 10 , the condition (9) for the positivity of the matrix $X$ reduces to the simple spectral condition: all eigenvalues of the matrix $X$ have to be nonnegative. But this is not true for the condition (10). Because the map $\Phi$ may be checked 1-positive, 2-positive etc; that condition does not ensure the positivity for all $n=1,2, \ldots \ldots \ldots$ To get a simple spectral condition for complete positivity, let us define the Choi-Jamiolkowsky matrix ([6], [10])

$$
C_{\Phi}:=\sum_{i, j=1}^{n_{1}}\left|e_{i}\right\rangle\left\langle e_{j}\right| \otimes \Phi\left(\left|e_{i}\right\rangle\left\langle e_{j}\right|\right)
$$

where $\left(e_{1}, e_{2}, \ldots \ldots \ldots, e_{n_{1}}\right)$ is an orthonormal basis in $C^{n_{1}}$. The Choi matrix $C_{\Phi} \in$ $M_{n_{1} n_{2}}(C)$ in (5) can also be written as

$$
C_{\Phi}=\sum_{i, j=1}^{n_{1}} E_{i j} \otimes \Phi\left(E_{i j}\right)
$$


Now, for $n$-positivity of the map $\Phi$ can be rewritten from condition (2) as follows:

$$
\left\langle\psi\left|C_{\Phi}\right| \psi\right\rangle \geq 0
$$

EXAMPLE 3. Let us consider a map $\Phi: M_{n} \rightarrow M_{n}$ defined by

$$
\Phi(X)=X^{T} \text {. }
$$

This map is trace preserving and trivially positive; since for $n=2$, we have

$$
X=\left(\begin{array}{ll}
E_{11} & E_{12} \\
E_{21} & E_{22}
\end{array}\right)=\left(\begin{array}{lll}
1 & 0 & 1 \\
0 & 0 & 0 \\
1 & 0 & 1
\end{array}\right)
$$

has spectrum $\{1,1,0,0\}$ and after performing the operation transposition the spectrum is not changed. But

$$
\begin{gathered}
\left(I_{2} \otimes \Phi\right)(X)=\Phi_{2}\left(\left(\begin{array}{ll}
E_{11} & E_{12} \\
E_{21} & E_{22}
\end{array}\right)\right) \\
=\left(\begin{array}{ll}
\Phi\left(E_{11}\right) & \Phi\left(E_{12}\right) \\
\Phi\left(E_{21}\right) & \Phi\left(E_{22}\right)
\end{array}\right)=\left(\begin{array}{ll|ll}
1 & 0 & 0 & 0 \\
0 & 0 & 1 & 0 \\
\hline 0 & 1 & 0 & 0 \\
0 & 0 & 0 & 1
\end{array}\right)
\end{gathered}
$$

has spectrum $\{-1,1,1,1\}$, shows that the matrix is indefinite. Hence, $\Phi$ is a positive map but not completely positive.

The following theorem is the generalized condition of completely positivity for all trivially positive maps:

THEOREM 5. (Choi theorem [17]) A map $\Phi: M_{n_{1}}(C) \rightarrow M_{n_{2}}(C)$ is completely positive if and only if its Choi matrix

$$
\begin{gathered}
C_{\Phi}=\sum_{i, j=1}^{n_{1}} E_{i j} \otimes \Phi\left(E_{i j}\right) \\
=\left(\begin{array}{c|c|ccc|c}
\Phi\left(E_{11}\right) & \Phi\left(E_{12}\right) & \ldots & \ldots & \ldots & \Phi\left(E_{1 n_{1}}\right) \\
\hline \Phi\left(E_{21}\right) & \Phi\left(E_{22}\right) & \ldots & \ldots & \ldots & \Phi\left(E_{2 n_{1}}\right) \\
\hline \vdots & & \ddots & & \vdots \\
\vdots & & & \ddots & & \vdots \\
\vdots & & & & \ddots & \vdots \\
\hline \Phi\left(E_{n_{1} 1}\right) & \Phi\left(E_{n_{1} 2}\right) & \ldots & \ldots & \ldots & \Phi\left(E_{n_{1} n_{1}}\right.
\end{array}\right)
\end{gathered}
$$

is positive semidefinite. 
DEFINITION 13. A completely positive map $\Phi: M_{n_{1}} \rightarrow M_{n_{2}}$ is said to be trace preserving if $\operatorname{Tr}(\Phi(X))=\operatorname{Tr}(X)$ for all $X \in M_{n_{1}}$.

A completely positive trace preserving map sends QI of a $n_{1}$-dimensional quantum system to a $n_{2}$ - dimensional quantum system. Thus, a completely positive trace preserving map is considered to be a QC.

DEFINITION 14. A QC $\Phi: M_{n_{1}} \rightarrow M_{n_{2}}$ is called unital if $\Phi\left(I_{n_{1}}\right)=I_{n_{2}}$. Otherwise, it will be said non-unital.

\section{Relation Between QCs and Doubly Stochastic Matrices.}

Positive maps acting on a space of nonnegative elements yield another nonnegative elements of a second space. Linear positive maps which are trace preserving and unital give rise to doubly stochastic matrices. Let $\left\{u_{1}, u_{2}, \ldots \ldots \ldots, u_{n}\right\}$ and $\left\{v_{1}, v_{2}, \ldots \ldots \ldots, v_{n}\right\}$ be to orthonormal basis in $C^{n}$. Then, we define

$$
D_{i j}=\left\langle u_{i}\left|\Phi\left(\left|v_{j}\right\rangle\left\langle v_{j}\right|\right)\right| u_{i}\right\rangle \text {. }
$$

Now, by definition $\Phi$ is positive if $D_{i j} \geq 0$. Taking sum over $i$, we obtain

$$
\sum_{i=1}^{n} D_{i j}=\sum_{i=1}^{n}\left\langle u_{i}\left|\Phi\left(\left|v_{j}\right\rangle\left\langle v_{j}\right|\right)\right| u_{i}\right\rangle=\operatorname{Tr}\left(\Phi\left(\left|v_{j}\right\rangle\left\langle v_{j}\right|\right)\right)
$$

Since $\Phi$ is trace preserving, we have $\operatorname{Tr}(\Phi(X))=\operatorname{Tr}(X)$. Using this fact, we get $\sum_{i=1}^{n} D_{i j}=\operatorname{Tr}\left(\Phi\left(\left|v_{j}\right\rangle\left\langle v_{j}\right|\right)\right)=1$, shows that $D_{i j}$ is a row stochastic Matrix. Again, taking sum over $j$, we obtain $\sum_{j=1}^{n} D_{i j}=\left\langle u_{i}\left|\sum_{j=1}^{n} \Phi\left(\left|v_{j}\right\rangle\left\langle v_{j}\right|\right)\right| u_{i}\right\rangle$.

Here, $\sum_{j=1}^{n} \Phi\left(\left|v_{j}\right\rangle\left\langle v_{j}\right|\right)=\Phi\left(\sum_{j=1}^{n}\left|v_{j}\right\rangle\left\langle v_{j}\right|\right)=\Phi\left(I_{n}\right)$, and for unitality of $\Phi, \Phi\left(I_{n}\right)=I_{n}$. Using these facts, we obtain

$$
\begin{aligned}
& \sum_{j=1}^{n} D_{i j}=\left\langle u_{i}\left|\sum_{j=1}^{n} \Phi\left(\left|v_{j}\right\rangle\left\langle v_{j}\right|\right)\right| u_{i}\right\rangle \\
& =\left\langle u_{i}\left|\Phi\left(I_{n}\right)\right| u_{i}\right\rangle=\left\langle u_{i}\left|I_{n}\right| u_{i}\right\rangle=1,
\end{aligned}
$$

shows that $D_{i j}$ is column stochastic. 
THEOREM 6. (Poon [24]) Let $\Phi: M_{n_{1}} \rightarrow M_{n_{2}}$ and $n \geq 1$. Then the followings are equivalent:

(i) $\Phi$ is $n$-positive

(ii) $\Phi$ is completely positive

(iii) The Choi matrix $C_{\Phi}$ is positive semidefinite

(iv) $\Phi$ admits an operator-sum representation

$$
\Phi(X)=\sum_{i=1}^{n} K_{i} X K_{i}^{*}
$$

If a map $\Phi$ is completely positive, then it is positive. Hence, a completely positive trace preserving unital map or unital QC, also gives rise to doubly stochastic matrices.

EXAMPLE 4. (Gagniuc [10]) Let us consider a family of Choi maps in $M_{n}$ defined by

$$
\Phi_{t}(X)=I_{n} \operatorname{Tr}(X)-t X, \quad t \leq 1
$$

Now, if $\frac{1}{k+1} \leq t \leq \frac{1}{k}$, then $\Phi_{\mathrm{t}}$ is $k$ positive but not completely positive. Hence, $\Phi_{\mathrm{t}}$ is completely positive if $t \leq \frac{1}{n}$. For $n=2$, the Choi matrix of $\Phi_{\mathrm{t}}$ is

$$
C_{\Phi}=\left(\begin{array}{cc|cc}
1-t & 0 & 0 & -t \\
0 & 1 & 0 & 0 \\
\hline 0 & 0 & 1 & 0 \\
-t & 0 & 0 & 1-t
\end{array}\right)
$$

which has the spectrum $\{1-2 t, 1,1,1\}$. Hence, $\Phi_{\mathrm{t}}$ is completely positive if $t \leq \frac{1}{2}$. Then the corresponding doubly stochastic matrix is

$$
D_{i j}=(1-t)\left(\begin{array}{ll}
1 & 0 \\
0 & 1
\end{array}\right)+t\left(\begin{array}{ll}
0 & 1 \\
1 & 0
\end{array}\right)
$$

That is , convex combination of two permutation matrices and hence a doubly stochastic matrix. For $t \leq \frac{1}{2}, \Phi_{\mathrm{t}}$ is a unital QC yielding a doubly stochastic matrix. By the motivation of the above remarkable results we present the following theorem in this article. We now generalize the condition whether a QC gives rise to a doubly stochastic matrix or not. 
THEOREM 7. Let $\Phi: M_{n_{1}} \rightarrow M_{n_{2}}$ be a completely positive map with Kraus operator $\left\{K_{i}\right\}$. Then the map $\Phi$ gives rise to doubly stochastic matrix if and only if $\sum_{i=1}^{n_{1}} K_{i}^{*} K_{i}=I$ and $\sum_{i=1}^{n_{1}} K_{i} K_{i}^{*}=1$.

Or, Let $\Phi: M_{n_{1}} \rightarrow M_{n_{2}}$ be a QC with Kraus operator $\left\{K_{i}\right\}$. Then the QC $\Phi$ gives rise to doubly stochastic matrix if and only if $\sum_{i=1}^{n_{1}} K_{i} K_{i}^{*}=1$.

Proof: We already know that every completely positive map $\Phi: M_{n_{1}} \rightarrow M_{n_{2}}$ can be expressed as

$$
\Phi(X)=\sum_{i=1}^{n_{1}} K_{i} X K_{i}^{*},
$$

Now, the map $\Phi$ gives rise to doubly stochastic matrix if and only if $\Phi$ is trace preserving and unital.

By definition, $\Phi$ is unital $\Leftrightarrow \Phi\left(I_{n_{1}}\right)=I_{n_{2}} \Leftrightarrow \sum_{i=1}^{n_{1}} K_{i} I_{n_{1}} K_{i}^{*}=I_{n_{2}} \Leftrightarrow \sum_{i=1}^{n_{1}} K_{i} K_{i}^{*}=$ $I_{n_{2}}$ and $\Phi$ is trace preserving $\Leftrightarrow \operatorname{Tr}(\Phi(X))=\operatorname{Tr}(X)$

$$
\begin{aligned}
& \Leftrightarrow \operatorname{Tr}\left(\sum_{i=1}^{n_{1}} K_{i} X K_{i}^{*}\right)=\operatorname{Tr}(X) \text { for all } X \in M_{n_{1}} \\
& \Leftrightarrow \operatorname{Tr}\left(\sum_{i=1}^{n_{1}} X K_{i}^{*} K_{i}\right)=\operatorname{Tr}(X) \text { for all } X \in M_{n_{1}} \\
& \Leftrightarrow \operatorname{Tr}\left(\sum_{i}^{n_{1}} X K_{i}^{*} K_{i}\right)-\operatorname{Tr}(X)=0 \text { for all } X \in M_{n_{1}} \\
& \Leftrightarrow \operatorname{Tr}\left(X\left(\sum_{i=1}^{n_{1}} K_{i}^{*} K_{i}-I_{n_{1}}\right)\right)=0 \text { for all } X \in M_{n_{1}} \\
& \Leftrightarrow\left(\sum_{i=1}^{n_{1}} K_{i}^{*} K_{i}-I_{n_{1}}\right)=0 ; \text { since } \operatorname{Tr}(X) \neq 0 \\
& \Leftrightarrow \sum_{i=1}^{n_{1}} K_{i}^{*} K=I_{n_{1}} .
\end{aligned}
$$




\section{CONCLUSION}

We studied the relationship between doubly stochastic matrices and majorization along with the structures of marginal doubly stochastic matrices in the Perfect-Mirsky region simply. We extended the Rivard-Mashreghi counterexample into a more general form for $n=5$ of the conjecture. Since the new conjecture for the characteristic region of doubly stochastic matrices given by Levick [18] contains a large part of the forbidden region for $n=5$ it needs a better analysis of the case $n \geq 5$. For a better idea of the location of eigenvalues in the Perfect-Mirsky region for $n \geq 5$ these counterexamples may be helpful to the readers for future research in the field. Every completely positive map has an operator-sum representation. Using these, we considered, in general, how can we get a doubly stochastic matrix corresponding to a QCs. The reader can go for further investigation into the relationship between doubly stochastic matrices and private QCs as well as the connection between spectra of doubly stochastic matrices and QCs. The connections between majorization, QCs, and private QCs are to be investigated. There are also many interesting connections between $\mathrm{QCs}$, private $\mathrm{QCs}$, and probability distribution.

\section{REFERENCES}

[1] Armandnejad, A., Akbarzadeh, F., and Mohammadi, Z.; Row and column-majorization on $M_{n, m}$, Linear Algebra and its applications, 437(2012), 1025-1032.

[2] Birkhoff, G.; Tresobservacionessobre el algebra lineal, Univ. Nac. Tucumán Rev. Se. A 5 (1946), 147-151.

[3] Bhuiyan, M. A. M.; Predicting Stochastic Volatility For Extreme Fluctuations In High Frequency Time Series. Open Access Theses \& Dissertations (2020), 2934.

[4] Cheng, Y., Carson, T. and Elgindi, M. B. M.; A Note on the Proof of the Perron-Frobenius Theorem, Applied Mathematics 3(11) (2012).

[5] Chruściński, D.; Positive maps, doubly stochastic matrices and new family of spectral conditions, Journal of Physics: Conference Series, IOP Publishing, 213(1) (2010), 012003 .

[6] Chruściński, D., and Kossakowski. A.; Spectral conditions for positive maps and entanglement witnesses. Journal of Physics: Conference Series. IOP Publishing, 284(1) (2011) 012017. 
[7] Das, H. K.; Affine Integral Quantization on a Coadjoint Orbit of the Poincaré Group in $(1+1)$-space-time Dimensions and Applications. Masters thesis, Concordia University (2015).

[8] Dahl, G.; Matrix majorization, Linear Algebra and Its Applications 288(1999), 53-73.

[9] Dmitriev, N. and Dynkin, E.; On characteristic roots of stochastic matrices", Izv. Akad. Nauk SSSR Ser. Mat., 10(2) (1946), 167-184.

[10] Gagniuc, P. A.; Markov Chains: From Theory to Implementation and Experimentation, John Wiley \& Sons (2017).

[11] Ito, H.; A new statement about the theorem determining the region of eigenvalues of stochastic matrices, Linear algebra and its applications, 267 (1997), 241-246.

[12] Jamiołkowski, A. (1972). Linear transformations which preserve trace and positive semidefiniteness of operators. Reports on Mathematical Physics, 3(4), 275-278.

[13] Karpelevič, F. I.; On characteristic roots of matrices with nonnegative coefficients, Uspehi Matem. Nauk (NS) 4.5 (33) (1949), 177-178.

[14] Kendall D. G., G. K. Batchelor, N. H. Bingham, W. K. Hayman, J. M. E. Hyland, G. G. Lorentz, H. K. Moffatt, W. Parry, A. A. Razborov, C. A. Robinson, P. Whittle.: Andrei Nikolaevich Kolmogorov (1903-1987), Bulletin of the London Mathematical Society, 22(1) (1990), 31-100.

[15] Kra, I., and Simanca, S. R.; On circulant matrices, Notices of the AMS 59(3) (2012), 368377.

[16] Kraus, K., Böhm, A. , Dollard, J. D. , and Wootters W. H.; States, effects and operations: fundamental notions of quantum theory, Springer (1983).

[17] Levick, J.; An Uncertainty Principle For Completely Positive Maps. Preprint arXiv:1611.06352 (2016).

[18] Levick, J.; New Methods for the Perfect-Mirsky Conjecture and Private Quantum Channels. Ph.D. thesis, University of Guelph (2015).

[19] Levick, J., Pereira, R. and Kribs, D. W.; The four-dimensional Perfect-Mirsky conjecture, Proceedings of the American Mathematical Society 143 (5) (2015), 1951-1956.

[20] Marshall, A. W., Olkin, I and Arnold, B. C.; Inequalities: theory of majorization and its applications, New York (1979).

[21] Mashreghi, J. and Rivard, R.; On a conjecture about the eigenvalues of doubly stochastic matrices, Linear and Multilinear Algebra 55(5) (2007), 491-498.

[22] Mehlum, M. S.; Doubly Stochastic Matrices and the Assignment Problem, MS thesis, (2012).

[23] Perfect, H., and Mirsky. L., Seffield; Spectral properties of doubly-stochastic matrices, Monatshefte für Mathematik 69 (1965): 35-57.

[24] Poon, Y. T.; Quantum operations, Summer School on Quantum Information Science Taiyuan University of Technology, Taiyuan, Shanxi, China, July 18 - 22 (2011).

[25] Prashanth B., K Nagendra Naik, KR Rajanna. A remark on eigen values of signed graph, Journal of Applied Mathematics, Statistics and Informatics, 15(1) (2019): 33-42. 
[26] Romanovsky, V; Recherchessur les chaines de Markoff, Acta mathematica 66(1) (1936): $147-251$.

[27] Samad, S.G. and W. N. Polyzou; Euclidean formulation of relativistic quantum mechanics of N particles, Physical Review C 103 (2021): 025203-22.

H. K. Das

Department of Mathematics,

University of Dhaka, Dhaka-1000, Bangladesh

Department of Mathematics,

Oklahoma State University, Stillwater, OK 74078, United States

e-mail: hkdas_math@du.ac.bd, haridas.das@okstate.edu

\section{Md. Kaisar Ahmed}

Department of Mathematics, University of Dhaka, Dhaka-1000, Bangladesh e-mail: kaisr.du94@gmail.com 